\title{
Non-Newtonian blood flow in human right coronary arteries: Steady state simulations ${ }^{* \dagger}$
}

\author{
Barbara M. Johnston and Peter R. Johnston ${ }^{\ddagger}$ \\ School of Science \\ Griffith University \\ Kessels Rd \\ Nathan \\ Queensland \\ Australia 4111 \\ Phone: 61-7-3875-7748 \\ Fax: 61-7-3875-7656 \\ E-mail: P.Johnston@.griffith.edu.au \\ Stuart Corney and David Kilpatrick \\ Department of Medicine \\ University of Tasmania \\ GPO Box 252-34 \\ Hobart \\ Tasmania \\ Australia 7001
}

August 4, 2003

Key Words: non-Newtonian, blood flow, right coronary artery, computational fluid dynamics

\footnotetext{
*Submitted as an original article to the Journal of Biomechanics (BIO/2002/002779 Version 3)

${ }^{\dagger}$ This work was funded in part by the Griffith University Research and Development Scheme and the National Health and Medical Research Council of Australia

${ }^{\ddagger}$ Corresponding author
} 


\begin{abstract}
This study looks at blood flow through four different right coronary arteries, which have been reconstructed from bi-plane angiograms. Five non-Newtonian blood models, as well as the usual Newtonian model of blood viscosity, are used to study the wall shear stress in each of these arteries at a particular point in the cardiac cycle. It was found that in the case of steady flow in a given artery, the pattern of wall shear stress is consistent across all models. The magnitude of wall shear stress, however, is influenced by the model used and correlates with graphs of shear stress versus strain for each model. For midrange velocities of around $0.2 \mathrm{~ms}^{-1}$, the models are virtually indistinguishable. Local and global non-Newtonian importance factors are introduced, in an attempt to quantify the types of flows where non-Newtonian behaviour is significant. It is concluded that, while the Newtonian model of blood viscosity is a good approximation in regions of midrange to high shear, it is advisable to use the Generalised Power Law model (which tends to the Newtonian model in those shear ranges in any case) in order to achieve better approximation of wall shear stress at low shear.
\end{abstract}




\section{Introduction}

The distribution of wall shear stress (WSS) in coronary arteries is thought to be a significant factor in the onset of coronary heart disease, one of the major causes of death in Western society. Over recent years, there has been controversy as to whether high, low or oscillating WSS is implicated in the development of atheroma (Fry, 1968; Caro et al., 1971; Giddens et al., 1990; Ku et al., 1985). In particular, within the coronary arteries atherosclerotic lesions have been associated with low and oscillating WSS (Krams et al., 1997; Kirpalani et al., 1999; Myers et al., 2001).

Despite the clinical importance of atherosclerosis in the coronary arteries, there have been relatively few studies in this area, probably because of the difficulty of studying blood flow in these arteries because of their complex geometries and small size. Most studies have been in the left main coronary bifurcation (Friedman et al., 1987; Asakura and Karino, 1990; He and $\mathrm{Ku}, 1996$; Perktold et al., 1998), although the right coronary artery (RCA) has been attracting some recent interest (Kirpalani et al., 1999; Myers et al., 2001; van Langenhove et al., 2000; Corney et al., 2001).

Another important aspect, in simulating blood flow and in assessing its impact on WSS, is to incorporate the nature of blood as a fluid. It is well known that blood behaves as a nonNewtonian fluid, particularly at low shear rates, that is, less than $100 \mathrm{~s}^{-1}$ (Pedley, 1980; Berger and Jou, 2000). Some models take blood flow to be Newtonian, on the assumption that the shear rates are greater than $100 \mathrm{~s}^{-1}$; however, the instantaneous shear rate over a cardiac cycle varies from zero to approximately $1000 \mathrm{~s}^{-1}$ in several large arterial vessels (Cho and Kensey, 1991).

In this paper, a number of non-Newtonian blood models, as well as the usual Newtonian model, are compared in a realistic simulation of blood flow in the RCAs of four different human subjects. These models are chosen because, even though there are many non-Newtonian models 
of blood rheology, no one model is universally accepted (Cho and Kensey, 1991; Ballyk et al., 1994; Cole et al., 2002; Ng et al., 2002; Misra and Ghosh, 2001).

The aims of this study are three-fold: firstly, to compare the various blood models, by studying WSS distributions in a particular artery and hence quantify the differences between models and judge their significance; secondly, to ascertain whether the use of the Newtonian blood model is appropriate over a wide range of shear rates and lastly, to study the variation in WSS in a particular artery as well as to compare WSS in the four different RCAs.

The remainder of this paper is organised as follows: Section 2 discusses the RCAs studied; Section 3, the various non-Newtonian blood models used; Section 4, the solution methodology; Section 5, steady state simulations; and Section 6, conclusions which can be drawn from this work.

\section{Right Coronary Arteries}

In this study, four different RCAs were studied. Three of the arteries (labelled A, B and D) exhibit typical coronary artery dimensions of 3-5 mm, whereas one artery (labelled C) is atypically large (a so-called 'ectatic' artery), with diameters varying between 5 and $7 \mathrm{~mm}$.

Images of these arteries were collected during routine angiography and were chosen because they showed no signs of atherosclerosis. The images were collected using a Siemens Bicor biplane angiogram machine and written to CD in a DICOM format. A pair of images taken at known angles, relative to one another, is shown in figure 1 for artery A. White crosses indicate the limits of the portion of the RCA which was reconstructed. The pairs of angiograms were taken at $80 \mathrm{~ms}$ intervals, during the following phases of the cardiac cycle: $\mathrm{B}$ and $\mathrm{D}$ were taken during systole; A was taken during early diastole and $\mathrm{C}$ during diastole.

Each pair of images was used to reconstruct the 3D geometry of the respective arteries, ignoring side-branches, using recently developed techniques based on centre-line extraction and 
radius determination (Corney et al., 2001). This technique determines the spatial position of the centre-line of the artery in three dimensions by detecting the centre-line in the two images and using the rotation angles embedded in the DICOM format.

Figure 2 shows the variation in arterial radius (calculated as a geometric mean of the radii for each view) as a function of distance along the artery. It is apparent that the arteries vary considerably in their geometry. For example, artery B has a more-or-less constant radius and artery D exhibits an almost uniform tapering. On the other hand, artery A shows two narrowings in the first $6 \mathrm{~cm}$ of the artery and then a bulging before returning to a reasonably constant radius. Finally, as mentioned above, artery $\mathrm{C}$ can be seen to have a larger radius than the other two arteries and appears to taper, then exhibit a slight narrowing, before returning to a constant radius.

Figure 2 also indicates the overall length of the arteries considered. The fact that artery $\mathrm{D}$ is significantly shorter than the other three arteries is related more to the reconstruction method used than to any physical variation in the arteries themselves.

\section{Non-Newtonian Blood Models}

One aspect of this paper involves consideration of the effects of various models for the nonNewtonian behaviour of blood on the WSS distribution in the coronary arteries. In total, five different non-Newtonian models are considered, as well as the simple Newtonian model. These models are summarised in Table 1, which also contains the input parameters for each model. The models have been created by various researchers (Cho and Kensey, 1991; Ballyk et al., 1994; Walburn and Schneck, 1976; Fung, 1993) by parameter fitting to experimental data for blood viscosity measured at certain shear rates. Good summaries for this aspect are contained in (Cho and Kensey, 1991) and (Ballyk et al., 1994), which both include a graph comparing apparent viscosity, for a range of shear rates and blood models, with experimental 
blood viscosity data from various different sources.

Figure 3(a) shows a plot of the apparent blood viscosity as a function of strain for each of these non-Newtonian models considered. At this point, it is worth discussing the strengths and weaknesses of these models. It is generally accepted that blood behaves as a Newtonian fluid for strains above $100 \mathrm{~s}^{-1}$ (Pedley, 1980; Berger and Jou, 2000). From figure 3(a), it can be seen that the Power Law model and Walburn-Schneck model (which is essentially another Power Law model) do not exhibit this behaviour as they predict decreasing viscosity at high strain. However, it has been suggested (Cho and Kensey, 1991) that the Power Law model could artificially mimic this behaviour by assuming a constant viscosity at strains above 226.5 $\mathrm{s}^{-1}$. This reference (Cho and Kensey, 1991) also lists alternative slightly different parameters for the Power Law model and these values were used in the study by Cole et al. (Cole et al., 2002). Additionally, the Walburn-Schneck model incorporates the haematocrit, $H$ (the value fraction of red blood cells in whole blood), but this simply serves to move the curve vertically and/or change its slope.

The Casson model can also take haematocrit into account (Fung, 1993), with the parameters given (obtained from data fitting) suggesting a value of $\mathrm{H}$ of $37 \%$; however, this yields a limiting viscosity at high shear slightly above the usual Newtonian value. The Carreau model does tend to the constant Newtonian case at high shear, but at well above the accepted $100 \mathrm{~s}^{-1}$ value. Other than that, this model appears to fit the experimental data quite well (Cho and Kensey, 1991).

Finally, the Generalised Power Law model (Ballyk et al., 1994) can be viewed as a general model for non-Newtonian blood viscosity. It encompasses the Power Law model at low strain, the Newtonian model at mid-range and high strain (from about $200 \mathrm{~s}^{-1}$ ) and has the Casson model as a special case (Ballyk et al., 1994). There is also close agreement between the Generalised Power Law and the Carreau model for low strain values (between 0.5 and $50 \mathrm{~s}^{-1}$ ). 


\section{Solution Methodology}

To study the behaviour of blood flow in the RCA, it must be assumed that blood can be represented by an incompressible fluid which is governed by the Navier-Stokes equations

$$
\rho \mathbf{v} \cdot \nabla \mathbf{v}=-\nabla \cdot \tau-\nabla P
$$

and the continuity equation

$$
\nabla \cdot \mathbf{v}=0
$$

where $\mathbf{v}$ is the $3 \mathrm{D}$ velocity vector, $P$ pressure, $\rho$ density and $\tau$ the shear stress term. Writing the Navier-Stokes equations in this form allows the various non-Newtonian blood models, described above and given in Table 1, to be included. It is also assumed that the artery walls are rigid and that the density of blood is $1050 \mathrm{~kg} \mathrm{~m}^{-3}$.

The governing equations are highly non-linear and must be solved numerically using the techniques of computational fluid dynamics. Here, these equations are solved using the finite volume method as implemented in the package CFD-ACE (CFDRC, Huntsville, Alabama, USA). The finite volume mesh is created based on the reconstructed coronary artery using our previously published techniques (Corney et al., 2001). Briefly, an inlet plane of the artery is meshed in 2D using quadrilaterals and this mesh is extruded along the centre-line of the artery to create a 3D mesh consisting of brick elements. An example mesh for artery A, consisting of a total of 62400 cells and 67769 nodes, is shown in figure 4. The inlet mesh, shown in figure 5 , contains 156 cells and 169 nodes.

Mesh independence has been confirmed by performing additional simulations using a greater number of nodes than those mentioned above (the 'original mesh'). Meshes were created with a larger number of nodes in the radial direction, resulting in a greater concentration of nodes near the wall of the artery, which, in theory, should allow more accurate determination of the wall shear stress. In all simulations performed on the more detailed mesh, maximum wall 
shear stresses differed from those on the original mesh by less than 1\%. Further, correlation coefficients for plots of the form shown in Figures 10 and 11, which compare WSS in the original and more detailed mesh, were 0.97 or higher. These considerations justify the choice of the original mesh described above.

Even though this study does not include the side-branches of the RCA, this restriction may not be significant as Myers et al. (Myers et al., 2001) have found that branch flows are not very important in determining WSS patterns in the main trunk of the RCA.

To solve the governing equations completely, a set of boundary conditions is required. Firstly, a no-slip condition is assumed along the walls of the artery. At the outlet, stress free conditions are applied and the gauge pressure is set to zero. Finally, a paraboloidal velocity profile, of the form $w(x, y)=v_{0}\left(1-\frac{x^{2}}{a^{2}}-\frac{y^{2}}{b^{2}}\right)$, with a given centre-line velocity, $v_{0}$, is specified at the inlet. The inlet is represented by an ellipse with semi-major axis $a$ and semiminor axis $b$, where it is assumed that the entrance plane is the $x-y$ plane. Although, such a profile is only correct for the Newtonian model of blood, it is a reasonable approximation for the non-Newtonian models as well. Each non-Newtonian model would have its own steady profile, which would be flatter at the centre-line and have higher velocity gradients near the wall. Such an approximation is required as it is difficult to obtain analytic representations for the steady velocity profiles for the non-Newtonian fluids considered, although they could be determined numerically.

Simple studies using cylindrical geometry have shown that the 'entrance length' (the distance in which a Newtonian profile changes to the non-Newtonian profile) is approximately three diameters at the lower velocities considered in this study, rising to nine diameters at mid-range velocities. The entrance length is reduced at higher velocities, as the flow is more Newtonian and so the parabolic inlet profile is a good approximation in this range. These entrance lengths are much smaller than the usual entrance lengths obtained for a uniform in- 
let flow. This approximation also allows consistent mass flow rates to be considered for all non-Newtonian blood models. In addition, a recent study of non-Newtonian versus Newtonian blood flow in the human RCA (Myers et al., 2001) has found that changes in inlet velocity profiles did not produce significant changes in WSS distributions for steady flow.

\section{$5 \quad$ Steady State Simulations}

For each of the four arteries, described in Section 2, and for each of the six blood viscosity models described in Table 1, a series of simulations was performed, where the centre-line velocity $v_{0}$, given in Section 4, was taken to be $0.02,0.05,0.1,0.2,0.5$ and $1.0 \mathrm{~ms}^{-1}$ respectively. These correspond to Reynolds numbers in the range 20-1000, depending on the artery.

A set of plots of WSS for each of the six viscosity models is presented for artery A in figures 6 to 8 , which correspond to centre-line velocities of $0.02,0.2$ and $1.0 \mathrm{~ms}^{-1}$ respectively. These pictures allow for a visual comparison of the WSS distributions to be made between the various models for a particular inlet velocity and also for various inlet velocities and a particular model.

Similar sets of plots were produced for each of the other three arteries, but these are not presented here. However, figure 9 contains plots of WSS in arteries B, C and D for the Generalised Power Law model with $v_{0}=0.2 \mathrm{~ms}^{-1}$. If these are studied in conjunction with the corresponding plot for artery A in figure 7(f), WSS can be compared in each of the arteries for the Generalised Power Law model.

Additional graphs, corresponding to these figures, were also made for each artery and inlet velocity for all six models. These graphs (see figures 10 and 11) were of WSS as a function of distance along a line drawn down the 'inside' wall of the artery (see figure 4, where the line used in artery $\mathrm{A}$ is the inside edge of the artery mesh). The line was drawn using the same point on the ellipses from which the mesh was constructed. Clearly, a different line will give different values of WSS. However, here it is the comparison between models that is of interest 
and investigation has shown that the conclusions reached below are still valid for other lines.

So the line graphs of figures 10(a), (b) and (c) show WSS, along the line mentioned above, corresponding to the plots in figures 6,7 and 8 and, in a similar fashion, the line graphs of figures 11(a), (b) and (c) correspond to the plots in figure 9.

\subsection{Descriptions of Wall Shear Stress Distributions}

It can be seen that each artery has its own distinct pattern of WSS, which is consistent across all models and similar for all inlet velocities. Only the magnitude of WSS varies between models and with changes in inlet velocities. For example:

(a) Artery A (see figures 6 to 8 and figure 10)

The WSS follows a complex pattern of peaks and troughs with a slight increase in magnitude at the end compared with the start of the artery. Stress peaks at about $6 \mathrm{~cm}$ down the artery and this is followed by a $4 \mathrm{~cm}$ region of low stress and then another peak. A major peak in WSS occurs near the end of the artery.

(b) Artery B (see figures 9(a) and 11(a))

This artery shows a pattern of alternating peaks and troughs of WSS, of similar magnitudes, with a greater number of peaks and troughs for the higher inlet velocities.

(c) Artery C (see figures 9(b) and 11(b))

Here a complex pattern of high and low WSS can be seen with up to eighteen peaks and troughs along the artery. WSS is low near the entrance and increases gradually over the length of the artery. There is a peak in WSS 'inside' the final bend of the artery.

(d) Artery D (see figures 9(c) and 11(c))

In this artery, there is a gradual increase in WSS over the length of the artery. There are alternating peaks and troughs of WSS, which increase in magnitude until near the end of the artery. This is consistent with the general tapering nature of the artery. 


\subsection{Non-Newtonian Importance Factor}

In order to quantify the effect of these non-Newtonian models on the WSS distributions, we now consider a concept introduced by Ballyk et al. (Ballyk et al., 1994), the non-Newtonian importance factor. They defined this as $\frac{\bar{\mu}_{e f f}}{\mu_{\infty}}$, where $\bar{\mu}_{e f f}$ is the effective viscosity characteristic of a particular flow and $\mu_{\infty}=0.0345 P$, the Newtonian value for viscosity. This was calculated for steady flow in a channel for the Generalised Power Law only and gave just one overall value for flow in a particular artery.

The idea is that the ratio gives an indication of the overall importance of non-Newtonian effects in the flow. We now introduce a variation of this ratio, where we use the values of apparent viscosity, $\mu$, which are produced as part of the solution process; that is, the ratio is calculated at any point in the artery, rather than just one overall figure as calculated by Ballyk et al.

We, therefore, define the local non-Newtonian importance factor, $I_{L}$, as

$$
I_{L}=\frac{\mu}{\mu_{\infty}}
$$

where $\mu$ is the viscosity (in Poise), given in Table 1 , and $\mu_{\infty}=0.0345 P$.

Clearly, $I_{L}=1$ for Newtonian flow and values which are significantly different from unity indicate regions of non-Newtonian flow. For example, Figure 12 shows a plot of $I_{L}$ for artery D for the Generalised Power Law with $v_{0}=0.05 \mathrm{~ms}^{-1}$. It can be seen that there are patches of non-Newtonian flow, particularly in the upper part of the artery and that the flow becomes more Newtonian near the end of the artery.

Calculating an average of these values would produce a figure similar in concept to that of Ballyk et al. but more representative of the actual flow in the artery. To facilitate the study of the relationship of this factor to WSS, the domain of calculation is restricted to the surface of the artery. Rather than simply averaging $I_{L}$ over the artery surface, the relative difference 
of each value of viscosity from the Newtonian value is averaged and expressed as a percentage. So the global non-Newtonian importance factor is defined as

$$
\bar{I}_{G}=\frac{1}{N} \frac{\left[\sum_{N}\left(\mu-\mu_{\infty}\right)^{2}\right]^{\frac{1}{2}}}{\mu_{\infty}} \times 100
$$

evaluated only at each of the $\mathrm{N}$ nodes on the surface of the artery. As above, $\mu$ is the viscosity (in Poise), given in Table 1 , and $\mu_{\infty}=0.0345 P$. For example, $\bar{I}_{G}=0.45$ for Figure 12.

It is obviously important to find a cut-off value of $\bar{I}_{G}$ above which the flow can be regarded as non-Newtonian. Table 2 shows the values of $\bar{I}_{G}$ for various centre-line velocities and models for artery A. For mid-range velocities, where $v_{0}=0.2 \mathrm{~ms}^{-1}$, the $\bar{I}_{G}$ values in Table 2 are all less than 0.25 . The plots of WSS corresponding to these values are presented in figure 7 and a graph corresponding to WSS on the inside wall of artery A (as discussed above) for each of the models is found in figure 10(b). These plots and graphs are all similar and therefore close to that produced by the Newtonian model and hence we would expect the value of $\bar{I}_{G}$, which simply represents the percentage difference of WSS from the Newtonian model, to be low. Another insight into the cut-off value from Newtonian to non-Newtonian can be gained from a study of the plots and graphs for the high velocity $v_{0}=1.0 \mathrm{~ms}^{-1}$. These are presented in figures 8 and 10(c). Here it can be seen that all the models produce WSS very close to the Newtonian values, except for the Power law and Walburn-Schneck models, which are clearly incorrect here (see Section 3). The $\bar{I}_{G}$ values, given in Table 2 , for these models are 0.59 and 0.31 respectively. This would tend to indicate that values of $\bar{I}_{G}<0.25$ could be regarded as near Newtonian, whereas for $\bar{I}_{G}$ above 0.25 the behaviour could be regarded as non-Newtonian. Tables similar to Table 2 were also produced for the other three arteries and after similar comparisons were made between $\bar{I}_{G}$ values and the WSS plots and graphs, it was concluded that a value of 0.25 would be an appropriate cut-off above which the behaviour could be regarded as non-Newtonian.

It should be noted that Ballyk et al. (Ballyk et al., 1994) suggested a cut-off value of 1.75 for their ratio $\frac{\bar{\mu}_{e f f}}{\mu_{\infty}}$, which was characteristic of the flow in the whole artery, not just the surface, 
and applied only to channel flow for the Generalised Power Law.

Based on the cut-off value of 0.25 suggested above, Table 2 indicates that for low centre-line velocities, such as $v_{0}=0.02 \mathrm{~ms}^{-1}$ and $v_{0}=0.05 \mathrm{~ms}^{-1}$, all models show significant non-Newtonian effects. This is consistent with plots such as those in figure 6 and graphs like those in figure 10(a). However, it should be noted that these differences are quite small in absolute terms, of the order of 0.1-0.2 Pa, even though they are significant as a percentage difference from the Newtonian values, which is the way $\bar{I}_{G}$ is defined. The question is whether such differences in WSS values are significant in biological terms. Since it is not yet clear exactly how low WSS is implicated in atherogenesis (Caro, 2001), it seems reasonable to be cautious in dismissing these variations as insignificant.

\subsection{Observations}

For a particular artery and a particular inlet velocity, there is a distinct pattern of WSS, which is consistent across all models (for example, see figures 6 to 8). This can be seen clearly in the graphs of WSS as a function of distance along a line drawn down the artery (see figure 10). One point to note here is, that since, for any particular line, inlet velocity and artery, the pattern of WSS is the same for the non-Newtonian and Newtonian models, this would tend to indicate that the entrance length (the distance for the non-Newtonian flow profile to become established) does not appear to have a major effect on WSS at the artery entrance.

The magnitude of WSS depends on the model used; for example, in artery A with centreline velocity $v_{0}=0.02 \mathrm{~ms}^{-1}$, the WSS produced by the Power Law model is higher than the Generalised Power Law and Carreau models, which have, in turn, higher values of stress than the Walburn-Schneck, Casson and lastly Newtonian models. As the inlet velocity becomes larger, the magnitude of WSS produced by the various models increases and becomes much more similar so that at a centre-line velocity of $0.2 \mathrm{~ms}^{-1}$, the models are almost indistinguishable. As the inlet velocity continues to increase, four of the models (Carreau, Casson, Generalised 
Power Law and Newtonian) continue to produce almost identical values of stress, while the Walburn-Schneck and Power Law models give much lower values of stress.

This relationship between the magnitudes of WSS produced by the various models, is consistent across all four arteries. For high inlet velocities, this result is not surprising since the Carreau, Casson and Generalised Power Law models all tend to the Newtonian model at high shear (see Section 3). In fact, the magnitude of WSS given by a particular model, relative to the other models, correlates with the graph of shear stress as a function of strain; see figure 3(b) which is, of course, related to the graph of apparent viscosity versus strain, figure 3(a), presented in Section 3.

For example, a centre-line velocity of $0.02 \mathrm{~ms}^{-1}$ leads to strain values in the range $20-40 \mathrm{~s}^{-1}$, where the stress for the various models can be seen to lie in the same order as described above. Strains around $300 \mathrm{~s}^{-1}$ are produced by a centre-line velocity of $0.2 \mathrm{~ms}^{-1}$ and it is clear that the stress is very similar for all models. As inlet velocity (and strain) increase, the Carreau, Casson, Generalised Power Law and Newtonian models are very similar with Walburn-Schneck and the Power Law models producing much lower values of stress (as can be seen in figures 8 and $10(\mathrm{c}))$.

\subsection{Comparison with Other Studies}

It is difficult to compare the results of this study with other work, as previous studies have generally involved flow through arterial stenoses rather than through a healthy RCA as in this study. However, this study is in general agreement with the findings of Ballyk et al. (Ballyk et al., 1994), for the Generalised Power Law during steady flow in a $45^{\circ}$ end-to-side anastomosis model of the femoral artery. They found that the character of the WSS patterns for Newtonian and non-Newtonian flow was very similar, while the magnitude of the non-Newtonian WSS was considerably greater than in the Newtonian case. This was for flow parameters where their value of non-Newtonian importance factor indicated that non-Newtonian effects would be 
significant; that is, it would correspond to our low inlet velocities, such as $0.02 \mathrm{~ms}^{-1}$ and 0.05 $\mathrm{ms}^{-1}$.

In addition, Cho and Kensey (Cho and Kensey, 1991) found that in non-stenosed regions of the coronary arterial casting, where they compared Newtonian flow with non-Newtonian flow (modified Powell-Eyring model), the WSS calculated with the Newtonian viscosity of blood, showed consistently smaller values than those obtained with non-Newtonian viscosity of blood. A comparison of the value of apparent viscosity predicted by the modified Powell-Eyring model (Cho and Kensey, 1991) and the Newtonian viscosity, indicates that for this shear rate (114 $\mathrm{s}^{-1}$ ), the Newtonian value is less than the non-Newtonian value, in much the same way as found for the non-Newtonian models considered in this study (see Section 5.3).

\section{Conclusions}

This study shows that there is considerable inter-individual variation in the geometry of the RCA. This can be seen, initially, in the graphs of arterial radius for arteries B and D, which were both taken during systole. These two arteries also have significantly different radii from arteries $\mathrm{A}$ and $\mathrm{C}$. While it may be argued that these differences are related to the fact that the angiograms were taken at different points in the cardiac cycle, this may not be the case, as for the RCA (unlike the left coronary artery), systolic blood flow is similar to that of diastolic blood flow (Kirpalani et al., 1999). Variations in curvature can be seen (in one plane) in figures 8 and 9. These geometric differences would be expected to lead to significant variations in arterial flow patterns and hence WSS distributions, as has been demonstrated here.

In the case of steady state flow through the right coronary arteries studied, it was found that each of the four different RCAs exhibited its own distinctive pattern of WSS and that the character of that pattern (that is, the position of peaks and troughs) remained relatively constant as the inlet velocity was increased. In general, WSS was found to be lower at the 
entrance to the artery than the end of the section studied, even though there were sometimes troughs of lower WSS throughout the artery.

Also, the pattern of WSS was found to be the same, in a particular artery, for a particular inlet velocity, no matter which of the five non-Newtonian models or the usual Newtonian model was used. The only difference was in the magnitude of WSS and these differences were significant for low inlet velocities and also, in the cases of the Walburn-Schneck model and the Power Law model, for high inlet velocities. At mid-range centre-line velocities (around 0.1-0.2 $\mathrm{ms}^{-1}$ ), the models are almost indistinguishable.

The differences in magnitude of WSS can be explained by the differences in the models themselves, as discussed in Section 3 and which can be seen in figure 3. This leads to the conclusions that (relative to the other models): the Power Law model over-estimates WSS at low inlet velocities and under-estimates it at high inlet velocities; the Walburn-Schneck model under-estimates WSS at high inlet velocities and the Newtonian model under-estimates WSS at low inlet velocities.

Final conclusions, in relation to the use of the various blood viscosity models for steady flow, are, therefore, that neither the Power Law nor Walburn-Schneck models should be used to model blood viscosity, for reasons detailed above, not even if the Power Law model is artificially constrained to the Newtonian value of viscosity above a certain strain value. The Newtonian model is certainly a good approximation in regions of mid-range to high shear, with the debate centering on whether the fact that it underestimates WSS in regions of low shear is biologically significant. A cautious approach would be to advise the use of the Generalised Power Law model. This model approximates WSS better than the Newtonian model for low inlet velocities and in regions of low shear, encompasses the Casson model as a special case, closely agrees with the Carreau model for low to mid-range shear and is effectively Newtonian at mid-range to high shear. 
Since it has been concluded that the non-Newtonian aspect of blood flow in the RCA is significant for low velocities and if this is taken together with the fact that there are times in a cardiac cycle where the flow rate is near zero, it is clearly worth performing transient simulations with a physiological flow profile and studying the resultant WSS distributions. One factor which would need to be considered in that case would be whether the use of a non-Newtonian model of blood viscosity in a pulsatile study is valid, given that the data on which such models are based is obtained under steady state conditions.

Clearly, there is an inverse relationship between the radius of the artery and WSS (based on conservation of mass). However, the curvature of the artery is also involved in this process and the connection between radius, curvature and WSS is subject to on-going examination. 


\section{References}

Asakura, T. and Karino, T., 1990. Flow patterns and spatial distribution of atherosclerotic lesions in human coronary arteries. Circulation Research, 66:1054-1066.

Ballyk, P. D., Steinman, D. A., and Ethier, C. R., 1994. Simulation of non-Newtonian blood flow in an end-to-end anastomosis. Biorheology, 31(5):565-586.

Berger, S. A. and Jou, L.-D., 2000. Flows in stenotic vessels. Annual Reviews of Fluid Mechanics, 32:347-382.

Caro, C. G., 2001. Vascular fluid dynamics and vascular biology and disease. Mathematical Methods in the Applied Sciences, 24:1311-1324.

Caro, C. G., Fitz-Gerald, J. M., and Schroter, R. C., 1971. Atheroma and arterial wall shear: observation, correlation and proposal of a shear dependent mass transfer mechanism for atherogenesis. Proceedings of the Royal Society of London, B177:109-159.

Cho, Y. I. and Kensey, K. R., 1991. Effects of the non-Newtonian viscosity of blood on flows in a diseased arterial vessel. Part 1: Steady flows. Biorheology, 28:241-262.

Cole, J. S., Watterson, J. K., and O'Reilly, M. J. G., 2002. Numerical investigation of the haemodynamics at a patched arterial bypass anastomosis. Medical Engineering and Physics, 24:393-401.

Corney, S., Johnston, P. R., and Kilpatrick, D., 2001. Cyclic flow patterns in human coronary arteries. In Computers in Cardiology, pages 21-24. IEEE, IEEE Press.

Friedman, M. H., Bargeron, C. B., Deters, O. J., Hutchins, G. M., and Mark, F. F., 1987. Correlation between wall shear and intimal thickness at a coronary artery branch. Atherosclerosis, 68:27-33. 
Fry, D., 1968. Acute vascular endothelial changes associated with increased blood velocity gradients. Circulation Research, 22:165-197.

Fung, Y. C., 1993. Biomechanics: Mechanical Properties of Living Tissues. Springer-Verlag, 2nd edition.

Giddens, D. P., Zarins, C. K., and Glagov, S., 1990. Response of arteries to near wall fluid dynamic behavior. Applied Mechanics Review, 43 (2):S98-S102.

He, X. and Ku, D. N., 1996. Pulsatile flow in the human left coronary artery bifurcation: average conditions. Transactions of the ASME, 118:74-82.

Kirpalani, A., Park, H., Butany, J., Johnston, K. W., and Ojha, M., 1999. Velocity and wall shear stress patterns in the human right coronary artery. Transactions of the ASME, 121:370-375.

Krams, R., Wentzel, J. J., Oomen, J. A. F., Vinke, R., H., J. C., Schuurbiers, de Feyter, P. J., Serruys, P. W., and Slager, C. J., 1997. Evaluation of endothelial shear stress and 3D geometry as factors determining the development of atherosclerosis and remodelling in human coronary arteries in vivo. Arteriosclerosis, Thrombosis and Vascular Biology, 17:2061-2065.

Ku, D., Giddens, D., Zarins, C., and Glagov, S., 1985. Pulsatile flow and atherosclerosis in the human carotid bifurcation: Positive correlation between plaque and low and oscillating shear stress. Arteriosclerosis, 5:293-302.

Misra, J. C. and Ghosh, S. K., 2001. A mathematical model for the study of interstitial fluid movement vis-a-vis the non-Newtonian behaviour of blood in a constricted artery. Computers and Mathematics with applications, 41:783-811. 
Myers, J. G., Moore, J. A., Ojha, M., Johnston, K. W., and Ethier, C. R., 2001. Factors influencing blood flow patterns in the human right coronary artery. Annals of Biomedical Engineering, 29:109-120.

Ng, E. Y. K., Siauw, W. L., and Chong, C. K., 2002. Simulation of oscillatory wall shear stress in channels with moving indentations. International Journal for numerical methods in engineering, 54:1477-1500.

Pedley, T. J., 1980. The fluid mechanics of large blood vessels. Cambridge University Press.

Perktold, K., Hofer, M., Rappitsch, G., Loew, M., Kuban, B. D., and Friedman, M. H., 1998. Validated computation of physiologic flow in a realistic coronary artery branch. Journal of Biomechanics, 31:217-228.

van Langenhove, G., Wentzel, J. J., Krams, R., Slager, C. J., Hamburger, J. N., and Serruys, P. W., 2000. Helical velocity patterns in a human coronary artery. Circulation, 102:e22-e24.

Walburn, F. J. and Schneck, D. J., 1976. A constitutive equation for whole human blood. Biorheology, 13:201-210. 


\section{Captions}

Table 1: Models of blood viscosity, $\mu$, given in Poise $P(1 P=0.1 P a s)$ as a function of strain, $\dot{\gamma}$, given in $\mathrm{s}^{-1}$

Table 2: Values of the global non-Newtonian importance factor, $\bar{I}_{G}$, for steady flow in artery $\mathrm{A}$

Figure 1: Angiograms for artery A

Figure 2: Arterial radius as a function of arc-length for each of the four arteries

Figure 3: Apparent viscosity or Shear stress as a function of strain for the various blood models

Figure 4: Mesh for artery A

Figure 5: Inlet mesh for artery A

Figure 6: Wall Shear Stress (in Pa) in artery A for $v_{0}=0.02 \mathrm{~ms}^{-1}$

Figure 7: Wall Shear Stress (in Pa) in artery A for $v_{0}=0.2 \mathrm{~ms}^{-1}$

Figure 8: Wall Shear Stress (in Pa) in artery A for $v_{0}=1.0 \mathrm{~ms}^{-1}$

Figure 9: Wall Shear Stress (in Pa) for $v_{0}=0.2 \mathrm{~ms}^{-1}$ in various arteries using the Generalised Power Law model

Figure 10: Wall Shear Stress (in Pa) along a line in artery A

Figure 11: Wall Shear Stress (in Pa) for $v_{0}=0.2 \mathrm{~ms}^{-1}$ along a line in various arteries

Figure 12: Plot of local non-Newtonian importance factor, $I_{L}$, in artery $\mathrm{D}$ for $v_{0}=0.05 \mathrm{~ms}^{-1}$ 
Newtonian Model

Carreau Model((Cho and Kensey, 1991))

Walburn-Schneck Model((Walburn and Schneck, 1976))

Power Law((Cho and Kensey, 1991))

Casson Model((Fung, 1993))

Generalised Power Law

Model((Ballyk et al., 1994))
EFFECTIVE VISCOSITY $\mu$

$\mu=0.0345 P$

$\mu=\mu_{\infty}+\left(\mu_{0}-\mu_{\infty}\right)\left[1+(\lambda \dot{\gamma})^{2}\right]^{\frac{n-1}{2}}$

where $\lambda=3.313 s, \mathrm{n}=0.3568$, $\mu_{0}=0.56 P$ and $\mu_{\infty}=0.0345 P$.

$\mu=C_{1} e^{C_{2} H}\left[e^{C_{4}\left(\frac{T P M A}{H^{2}}\right)}\right](\dot{\gamma})^{-C_{3} H}$

where $C_{1}=0.00797, C_{2}=0.0608$, $C_{3}=0.00499, C_{4}=14.585 \mathrm{lg}^{-1}$, $H=40 \%$ and TPMA $=25.9 g^{-1}$

$\mu=\mu_{0}(\dot{\gamma})^{n-1}$

where $\mu_{0}=0.035$ and $n=0.6$.

$\mu=\left[\left(\eta^{2} J_{2}\right)^{\frac{1}{4}}+2^{-\frac{1}{2}} \tau_{y^{\frac{1}{2}}}\right]^{2} J_{2}^{-\frac{1}{2}}$

where $|\dot{\gamma}|=2 \sqrt{ } J_{2}$,

$\tau_{y}=0.1(0.625 H)^{3}$ and $\eta=\eta_{0}(1-H)^{-2.5}$ with $\eta_{0}=0.012 P$ and $H=0.37$.

$\mu=\lambda|\dot{\gamma}|^{n-1}$

$\lambda(\dot{\gamma})=\mu_{\infty}+\Delta \mu \exp \left[-\left(1+\frac{|\dot{\gamma}|}{a}\right) \exp \left(\frac{-b}{|\dot{\gamma}|}\right)\right]$

$n(\dot{\gamma})=n_{\infty}-\Delta n \exp \left[-\left(1+\frac{|\dot{\gamma}|}{c}\right) \exp \left(\frac{-d}{|\dot{\gamma}|}\right)\right]$

where $\mu_{\infty}=0.035, n_{\infty}=1.0$, $\Delta \mu=0.25, \Delta n=0.45, a=50$, $b=3, c=50$ and $d=4$.

Table 1: 


\begin{tabular}{|c|c|c|c|c|c|c|}
\hline \multirow{2}{*}{ Blood Model } & \multicolumn{5}{|c|}{ Centre-line Velocities $\left(\mathrm{ms}^{-1}\right)$} \\
\cline { 2 - 7 } & 0.02 & 0.05 & 0.1 & 0.2 & 0.5 & 1.0 \\
\hline \hline Power Law & 1.72 & 0.90 & 0.46 & 0.24 & 0.44 & 0.59 \\
\hline Generalised Power Law & 0.93 & 0.47 & 0.21 & 0.10 & 0.10 & 0.04 \\
\hline Carreau & 0.87 & 0.49 & 0.32 & 0.21 & 0.13 & 0.08 \\
\hline Walburn-Schneck & 0.74 & 0.45 & 0.27 & 0.13 & 0.20 & 0.31 \\
\hline Casson & 0.37 & 0.27 & 0.22 & 0.18 & 0.15 & 0.13 \\
\hline
\end{tabular}

Table 2: 


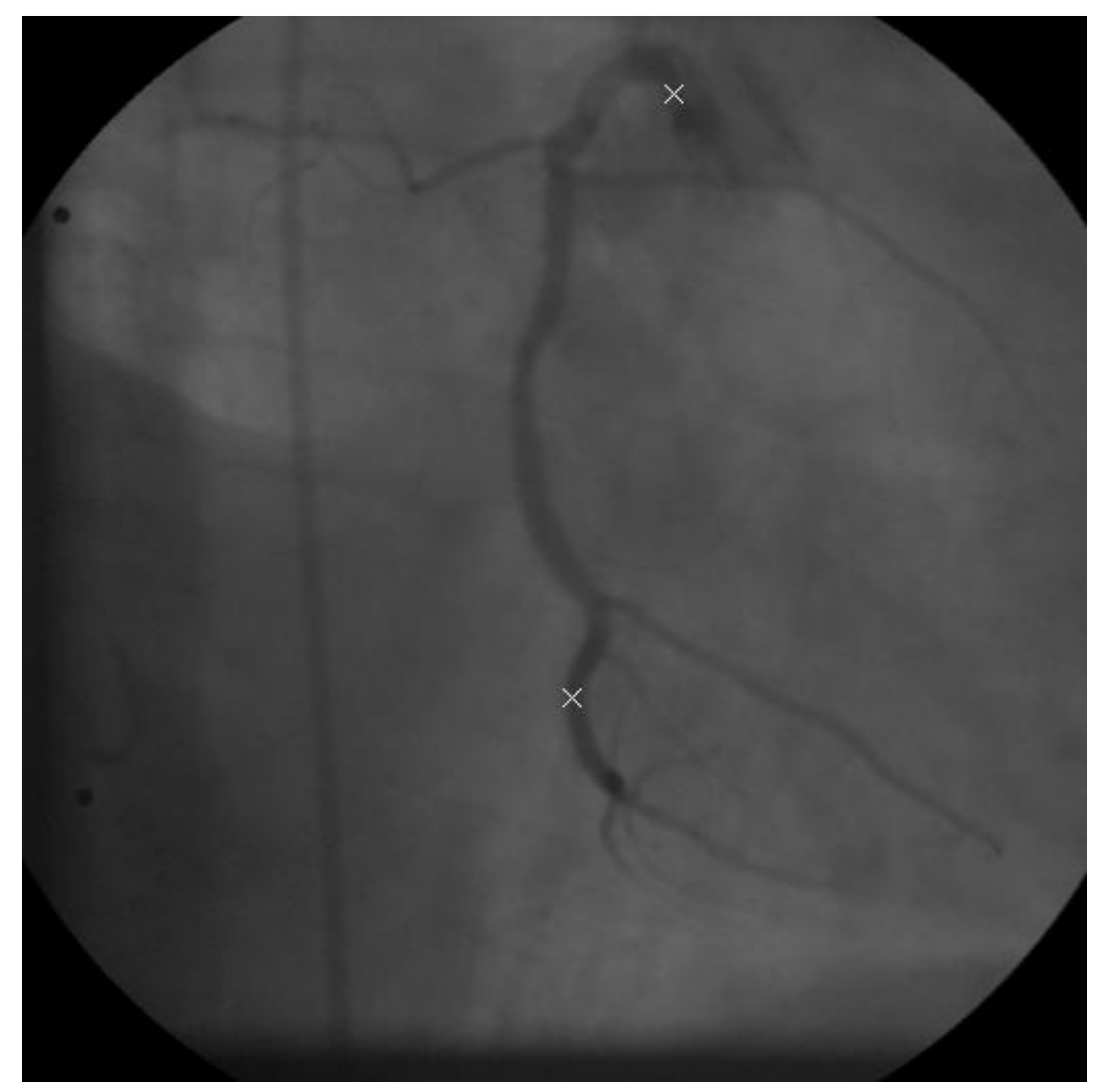

(a) Frame 15

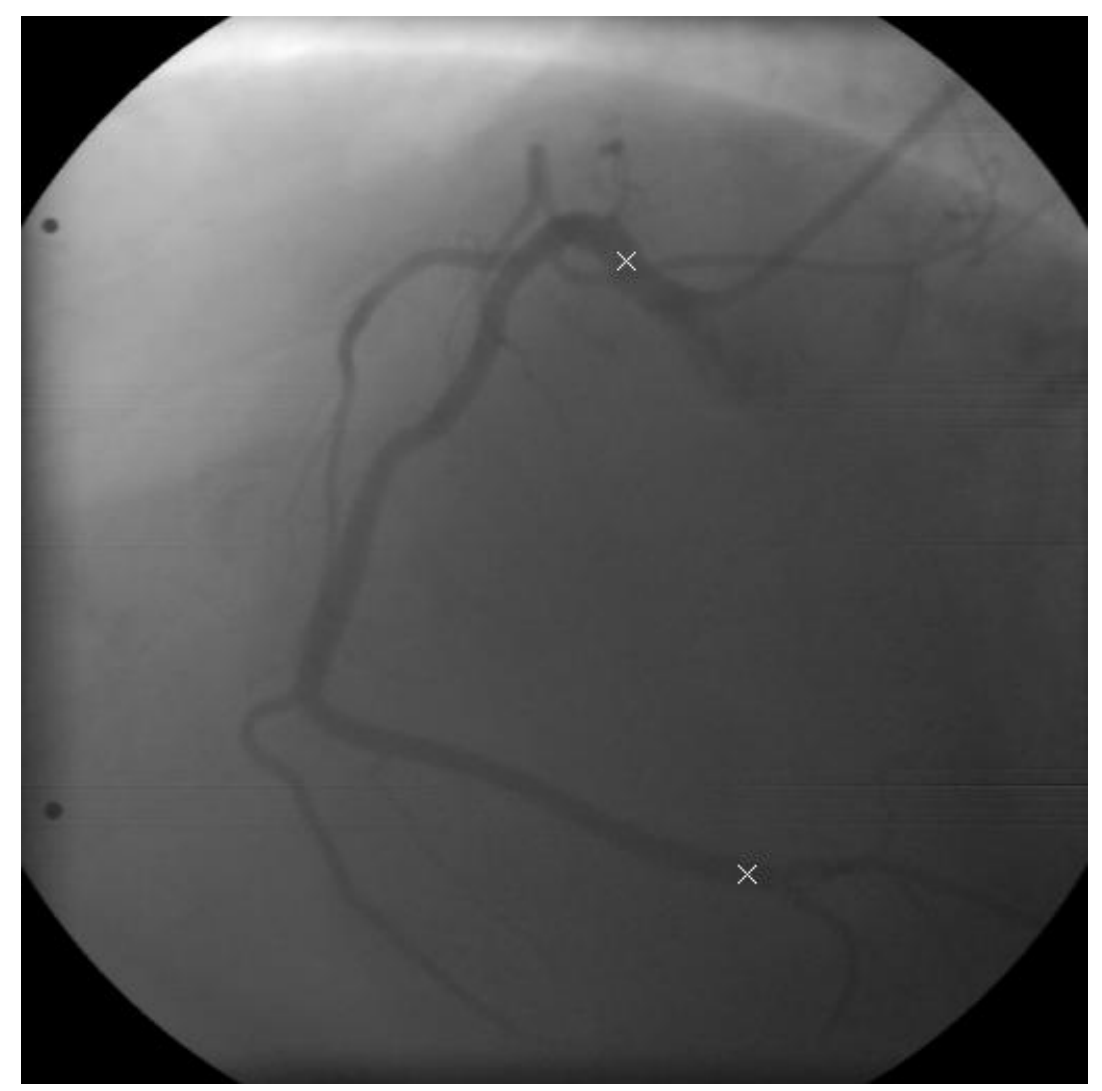

(b) Frame 16

Figure 1: 


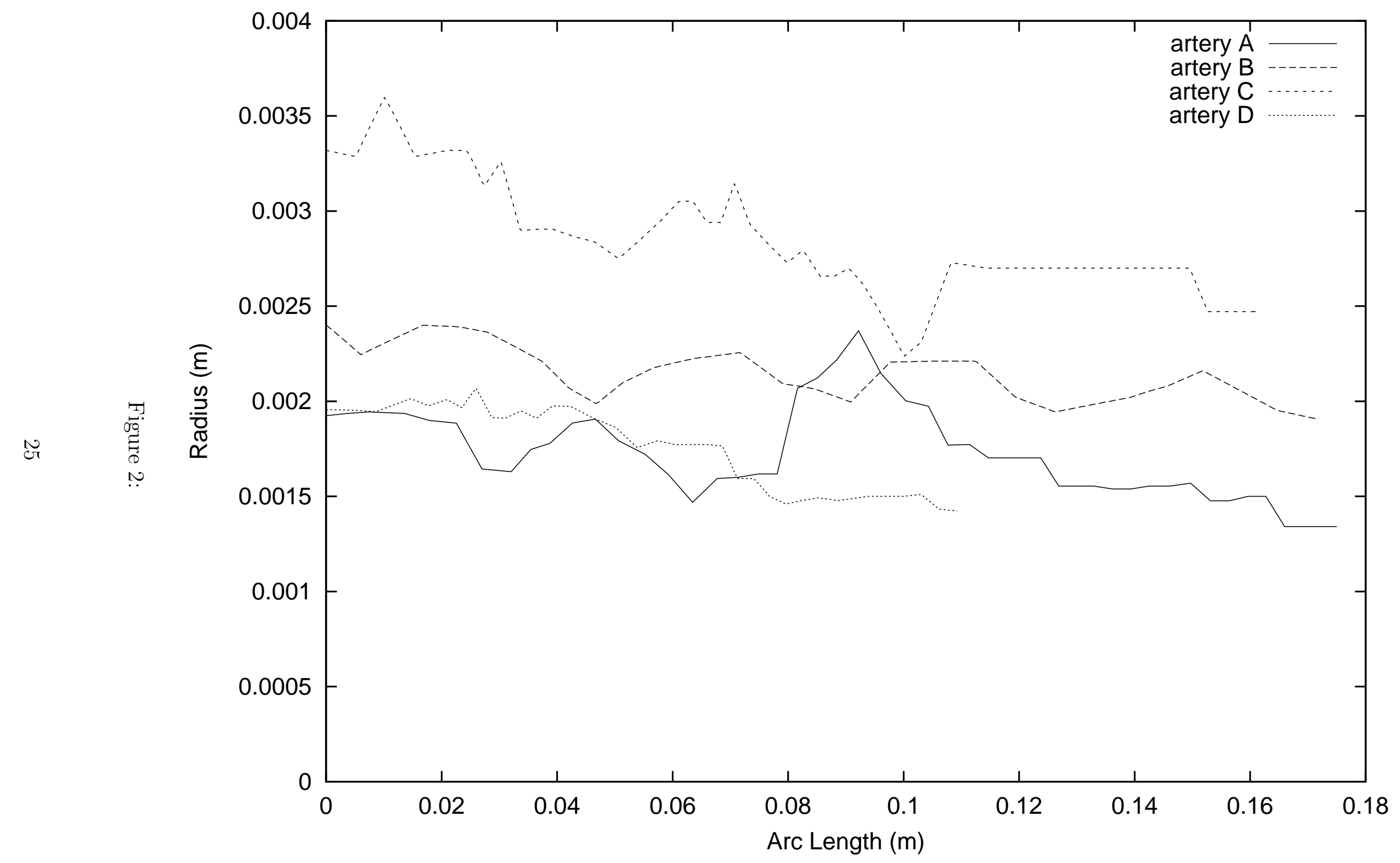




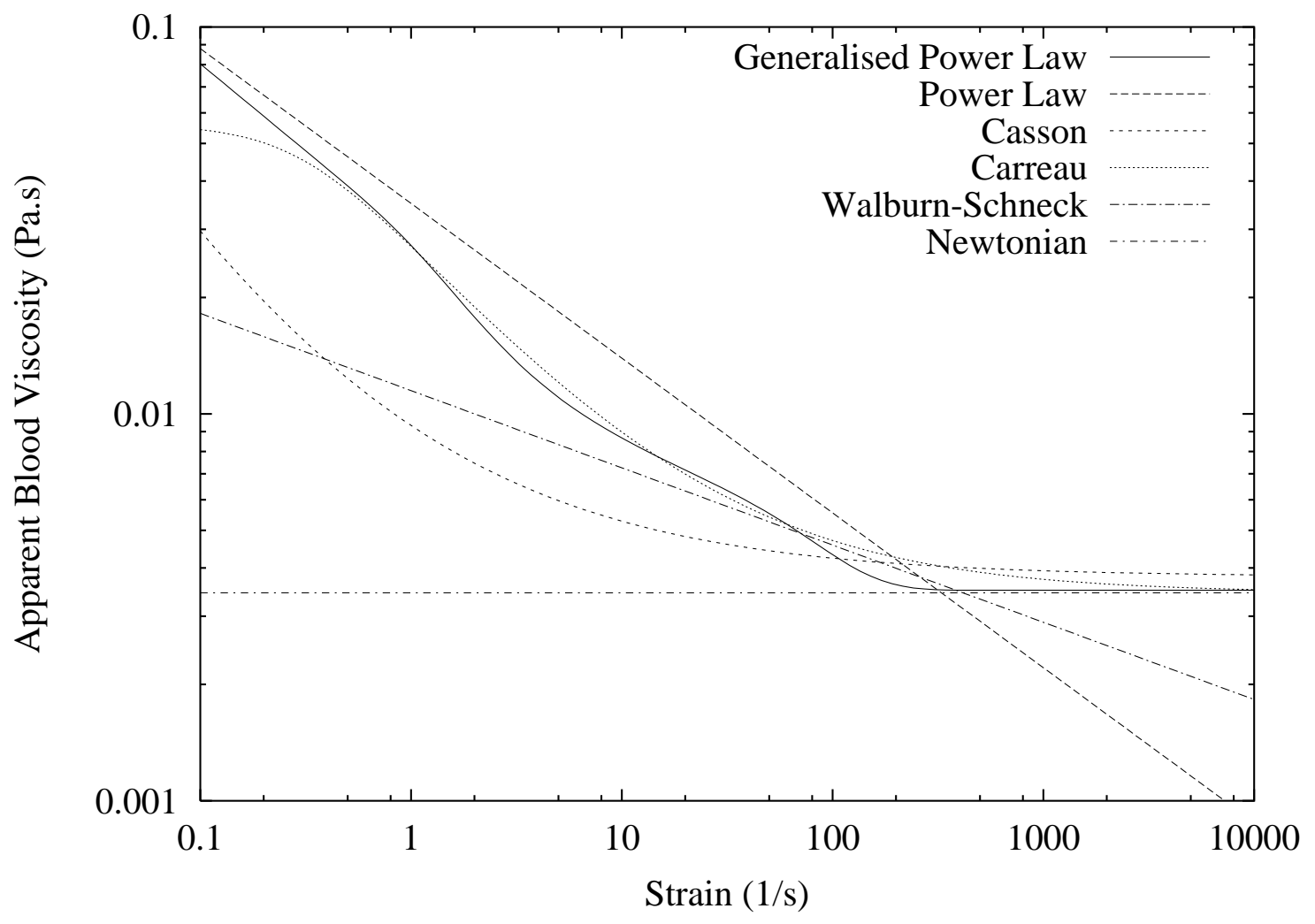

(a) Viscosity

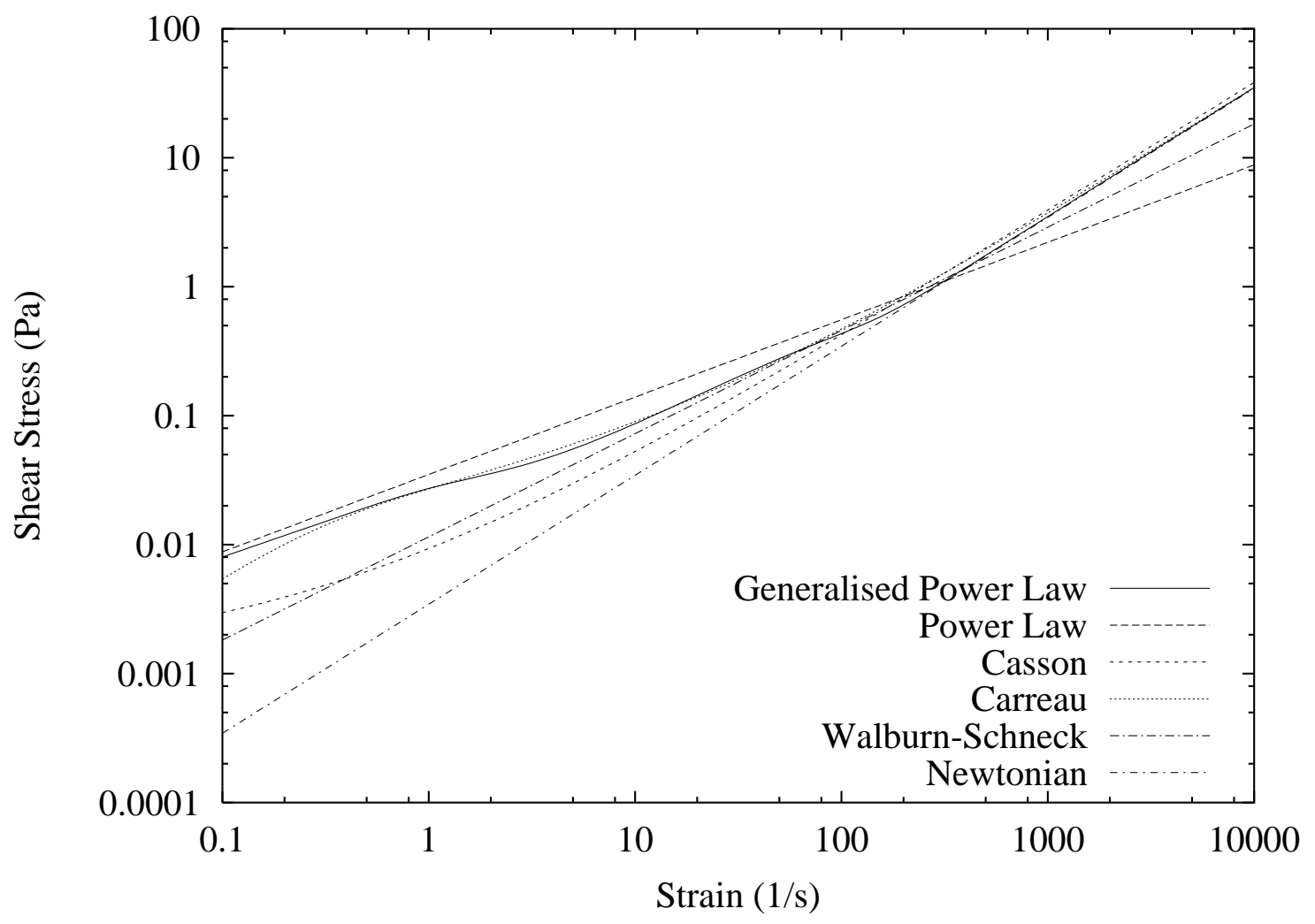

(b) Shear Stress

Figure 3: 


$$
\text { L }
$$




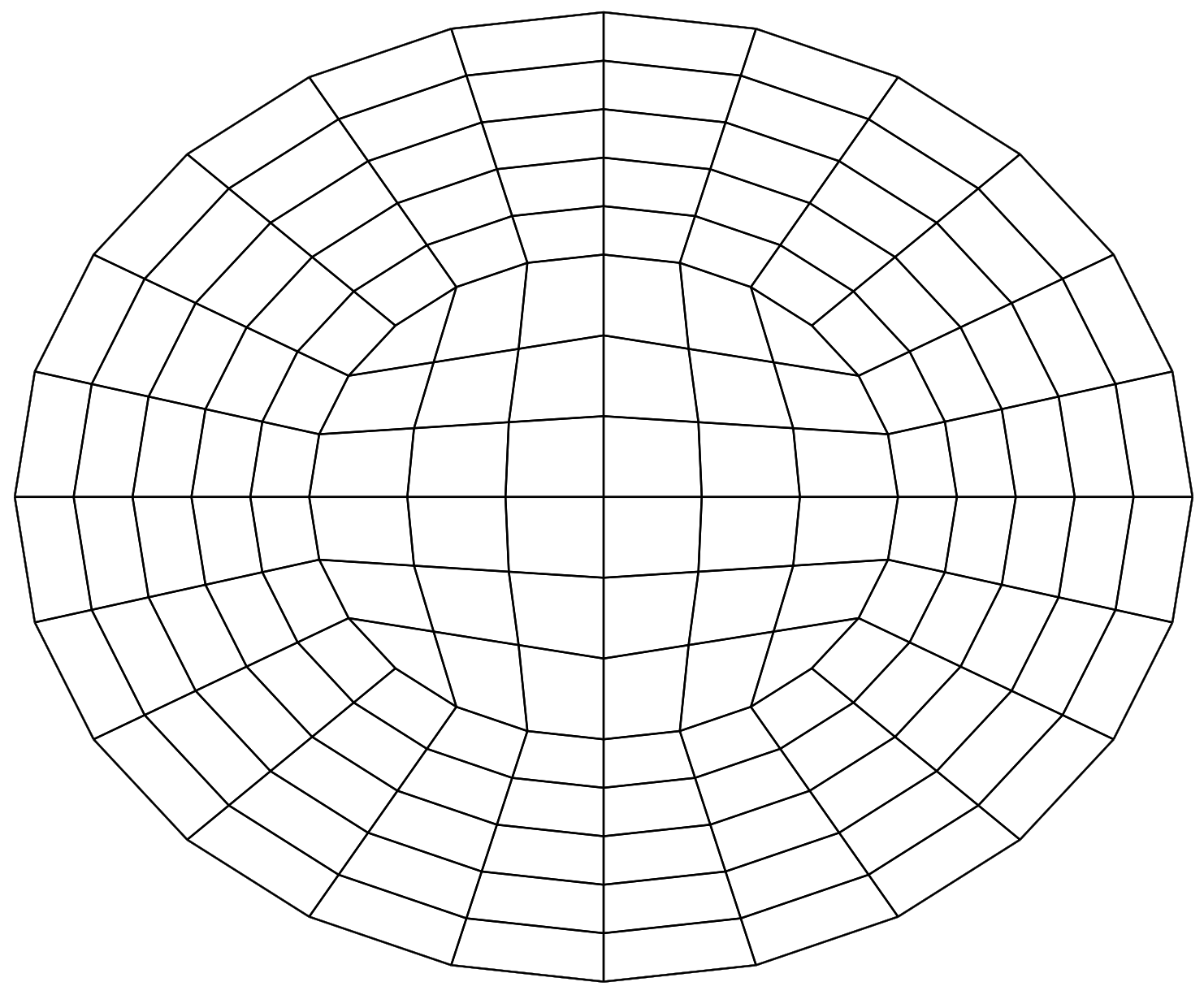

Figure 5: 


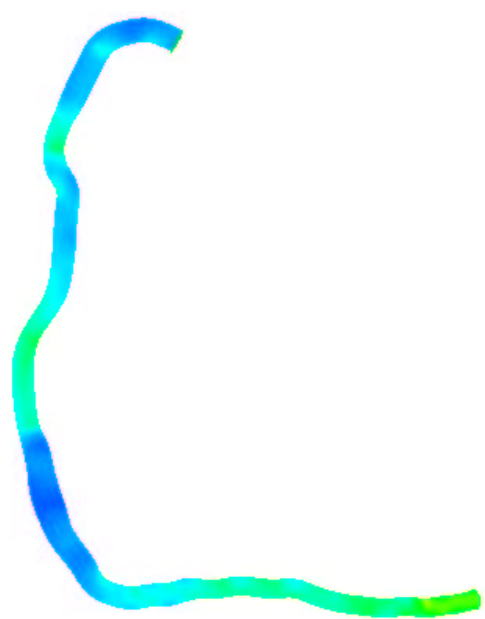

(a) Newtonian

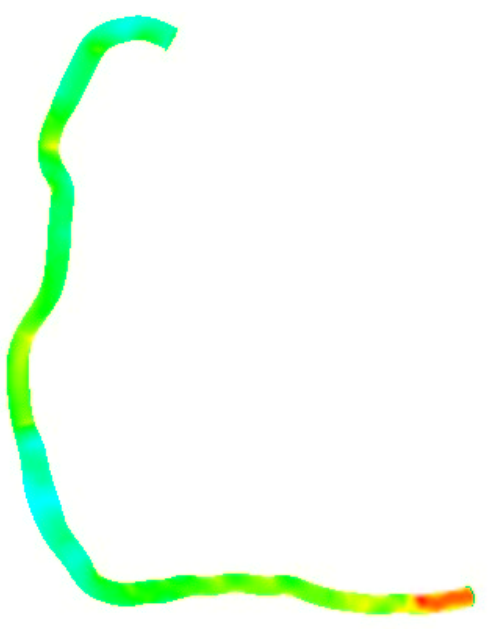

(c) Carreau

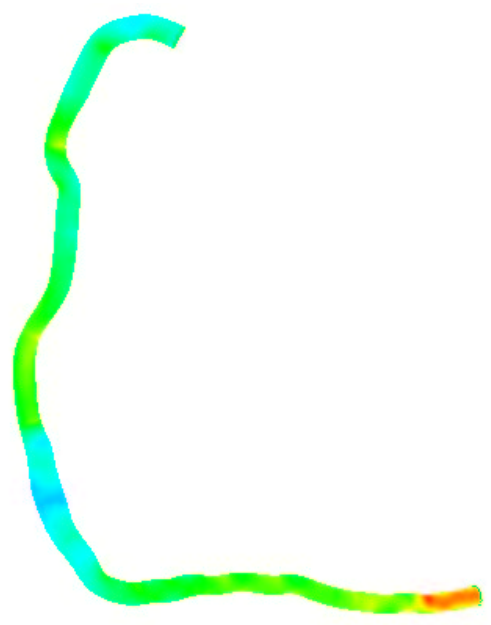

(e) Walburn-Schneck

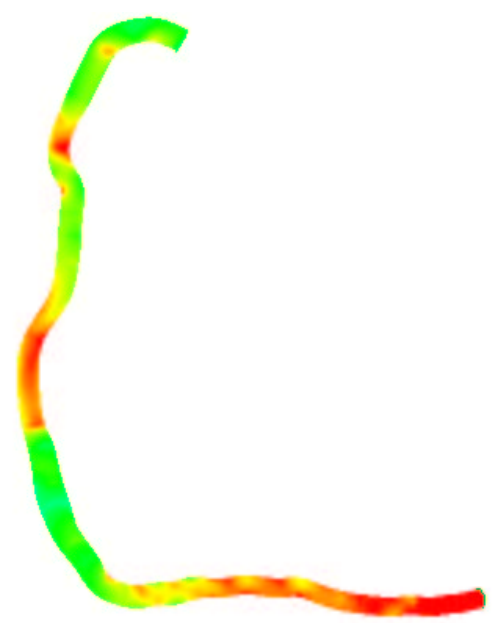

(b) Power Law

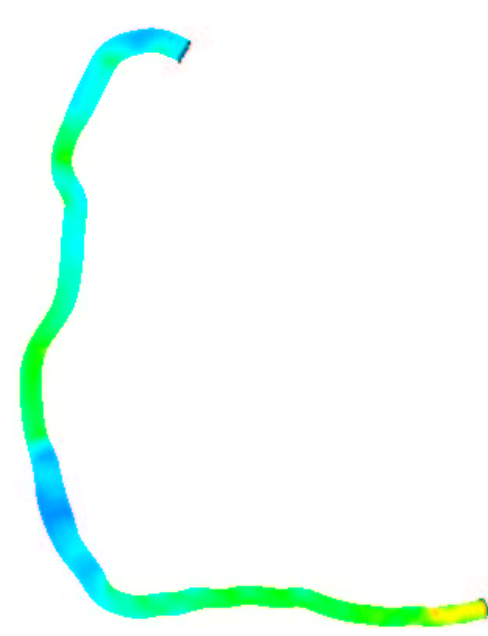

(d) Casson

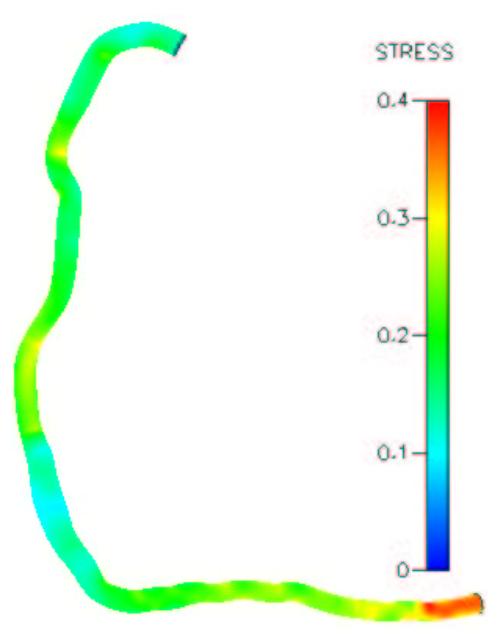

(f) Generalised Power Law

Figure 6: 


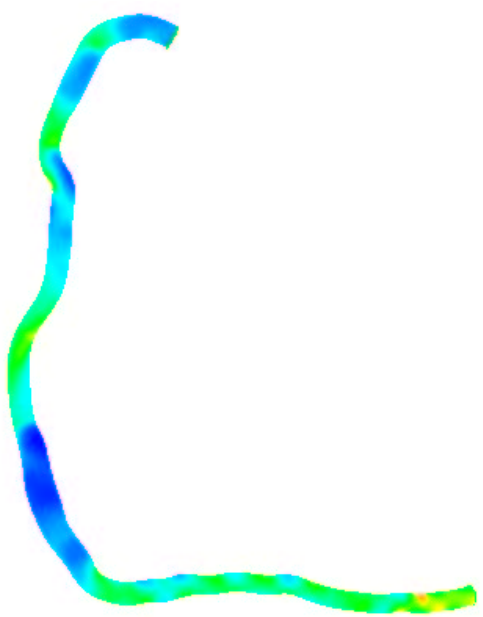

(a) Newtonian

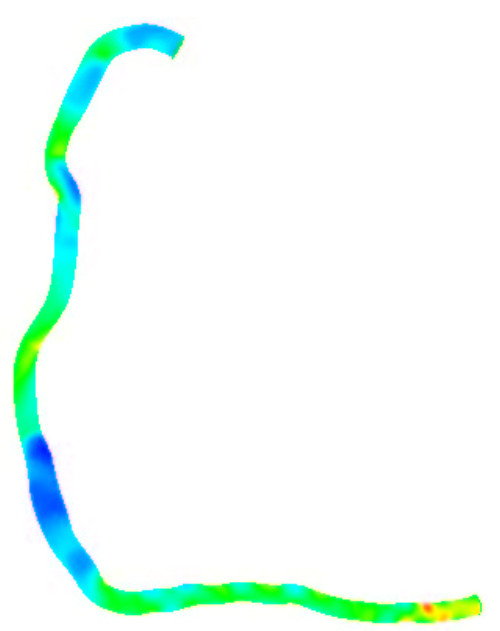

(c) Carreau

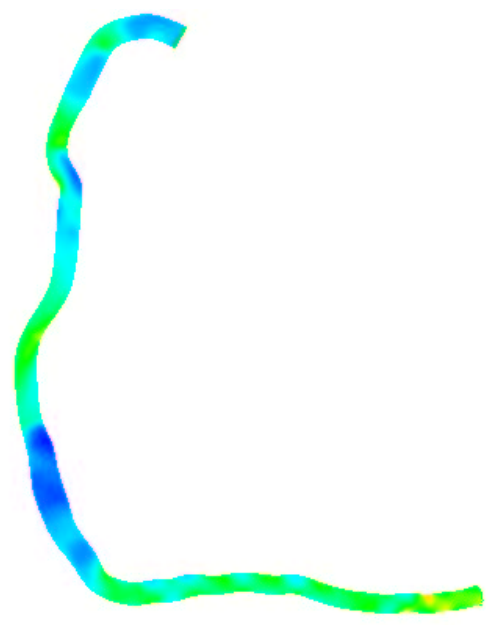

(e) Walburn-Schneck

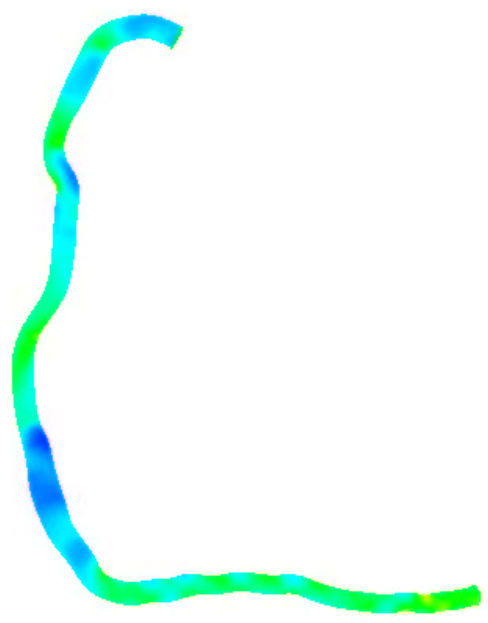

(b) Power Law

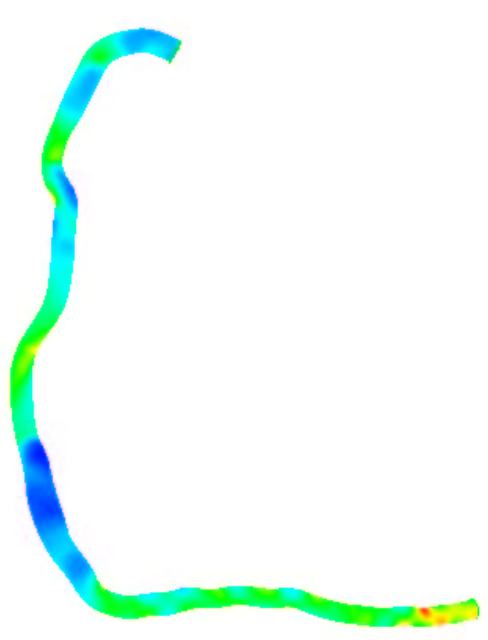

(d) Casson

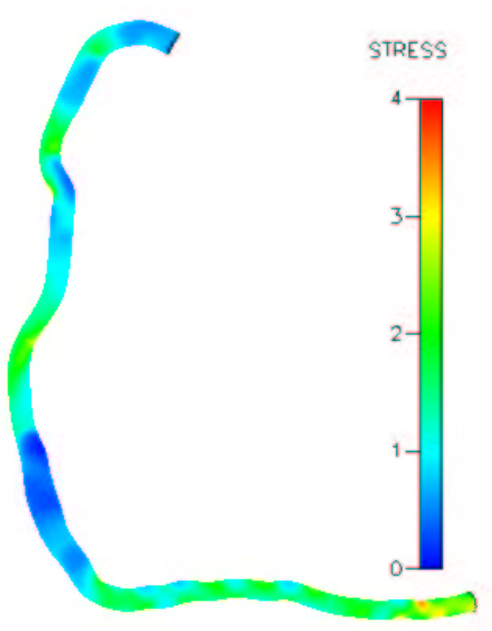

(f) Generalised Power Law

Figure 7: 


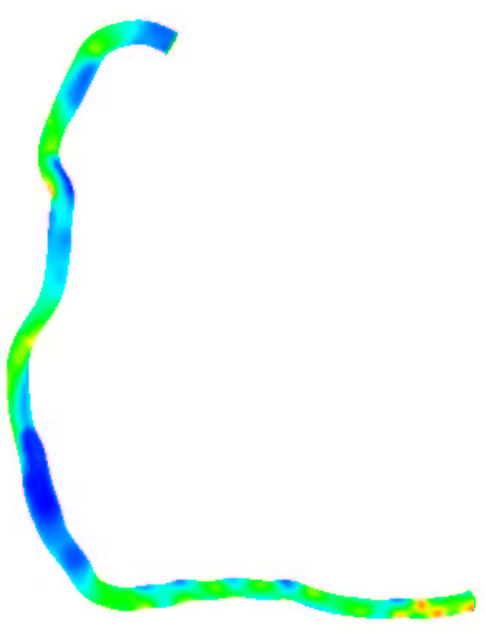

(a) Newtonian

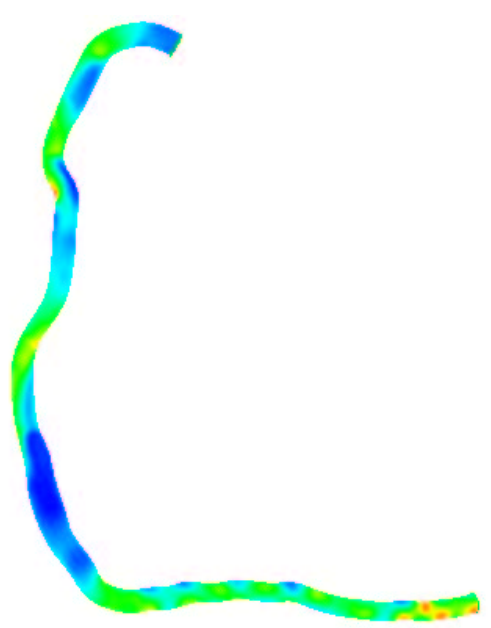

(c) Carreau

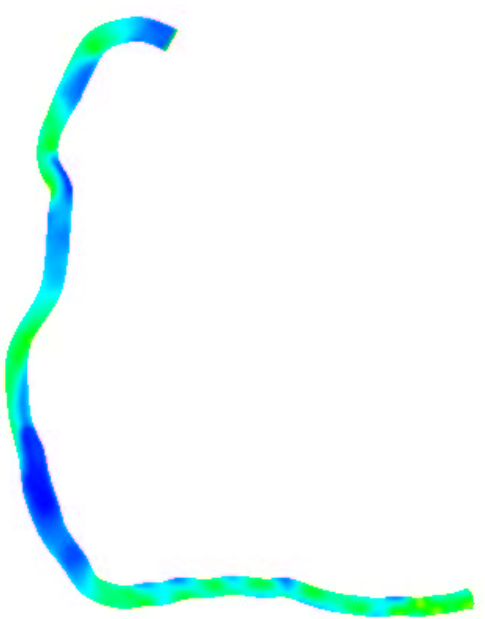

(e) Walburn-Schneck

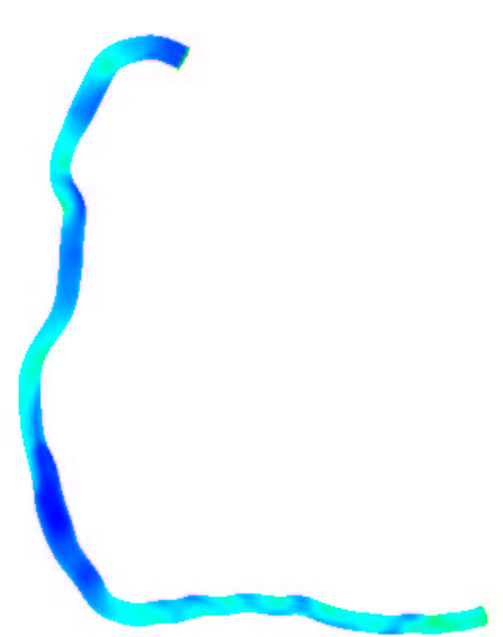

(b) Power Law

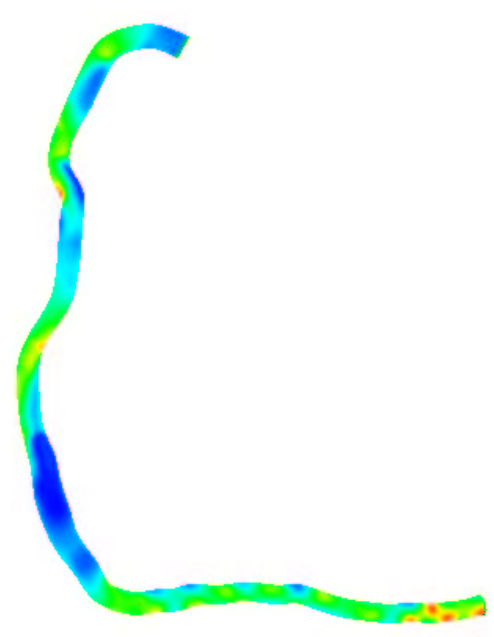

(d) Casson

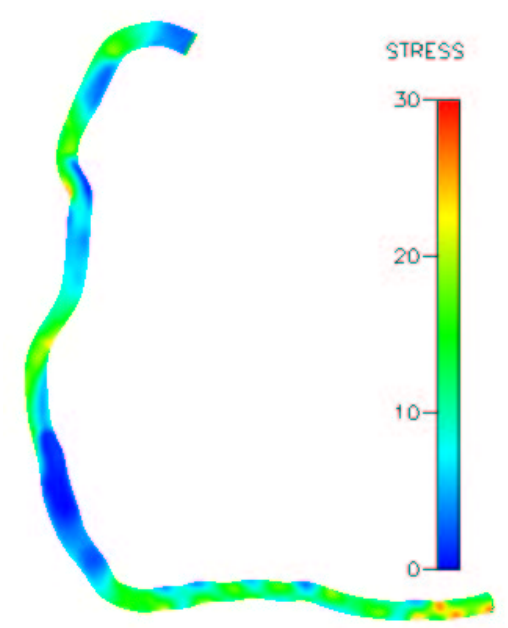

(f) Generalised Power Law

Figure 8: 


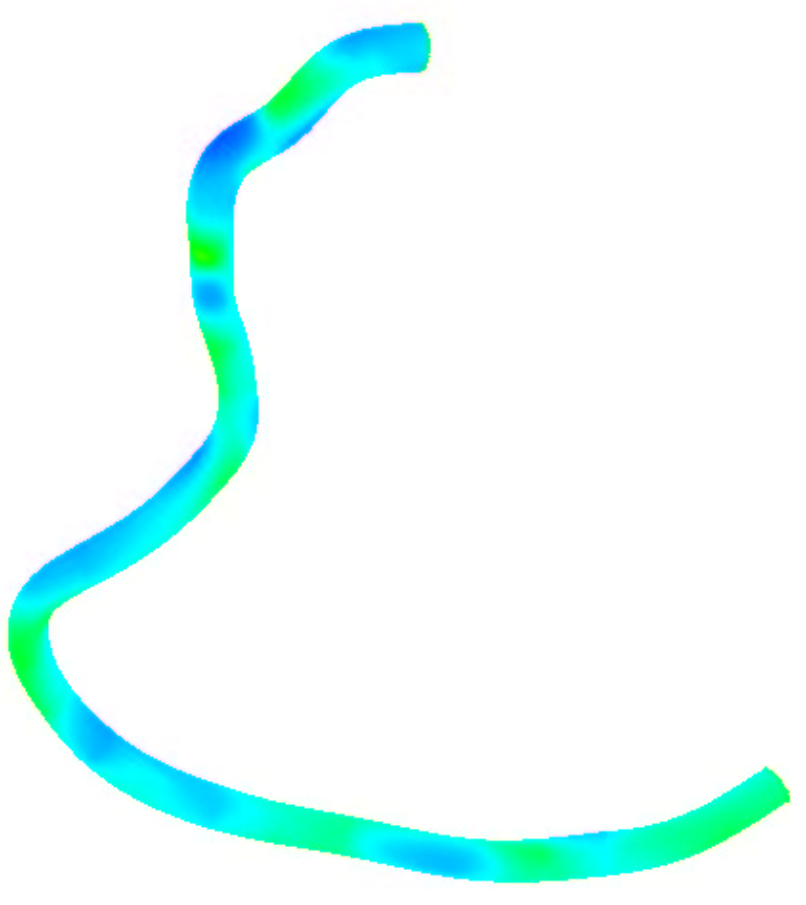

(a) Artery B

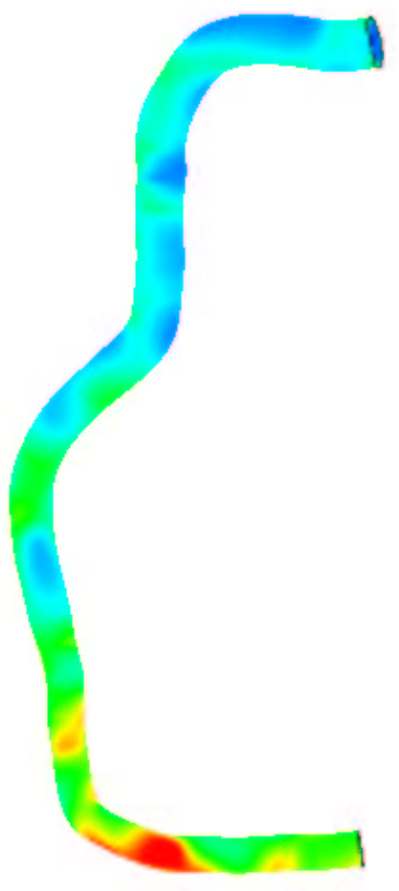

(c) Artery D

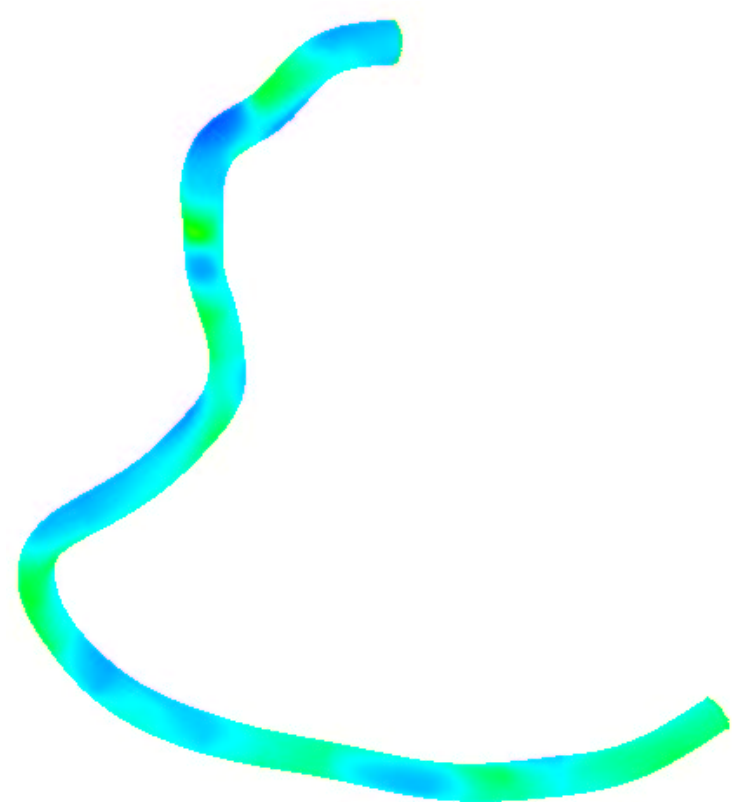

(b) Artery C

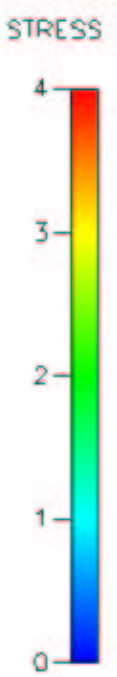

Figure 9: 


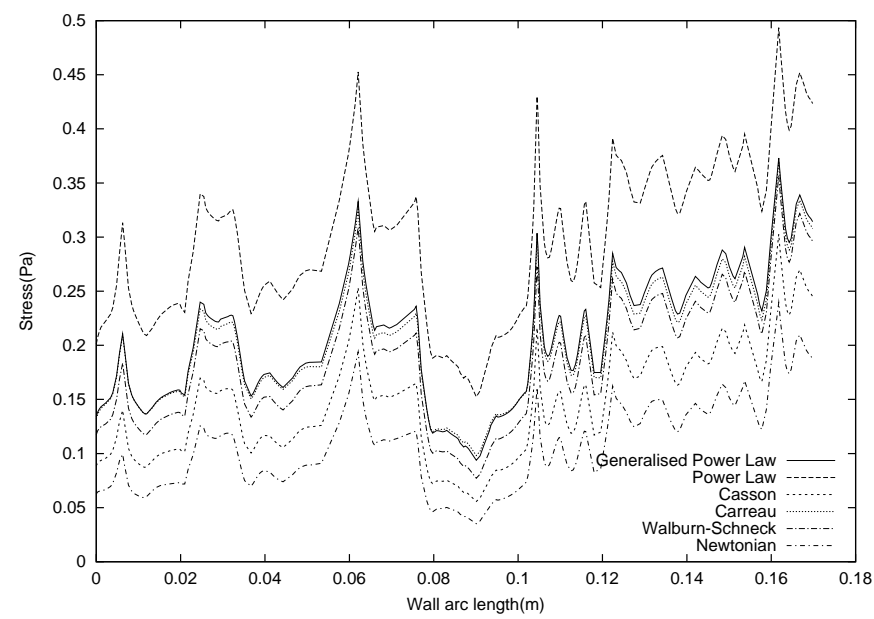

(a) $v_{0}=0.02 \mathrm{~ms}^{-1}$

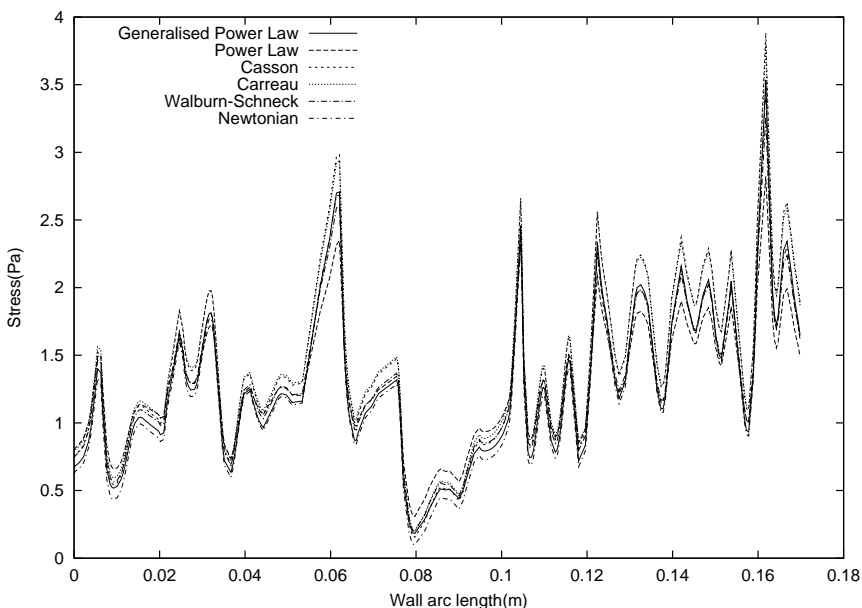

(b) $v_{0}=0.2 \mathrm{~ms}^{-1}$

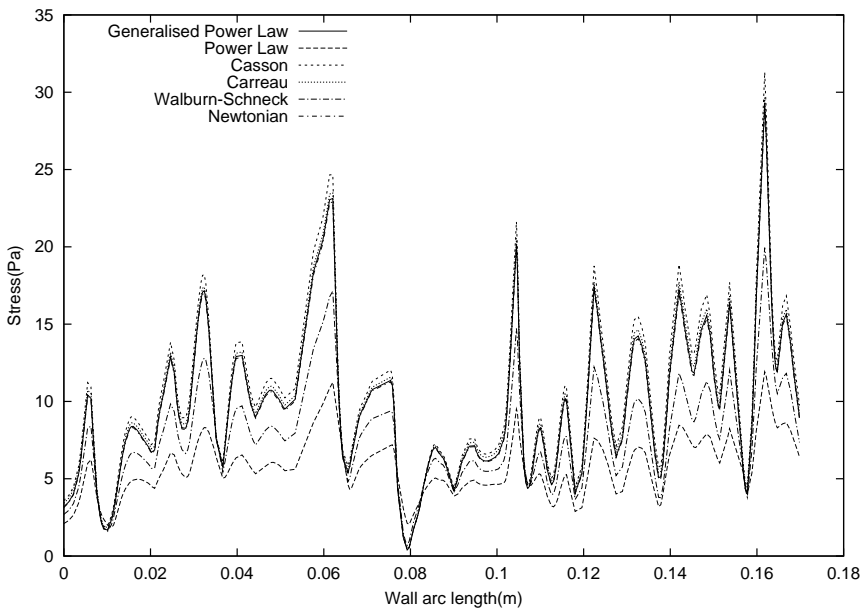

(c) $v_{0}=1.0 \mathrm{~ms}^{-1}$

Figure 10: 


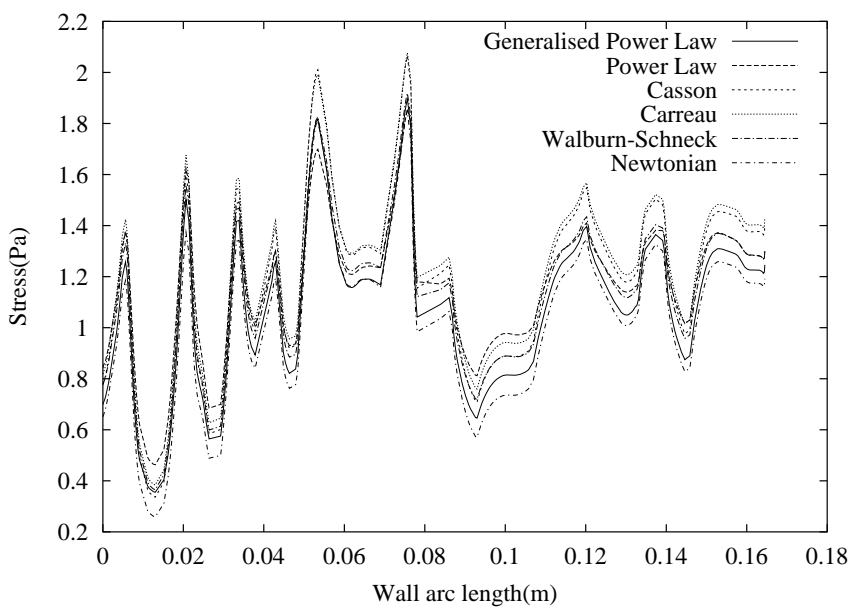

(a) artery B

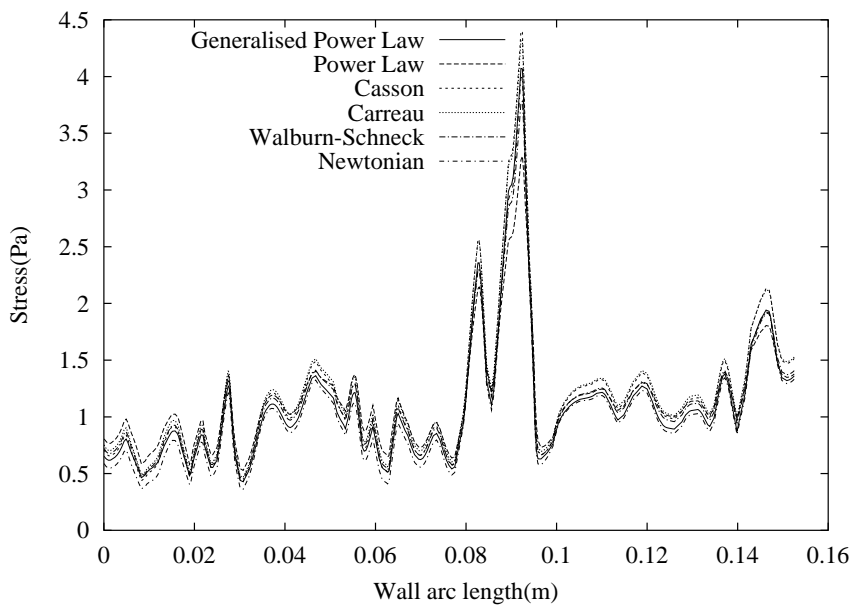

(b) artery C

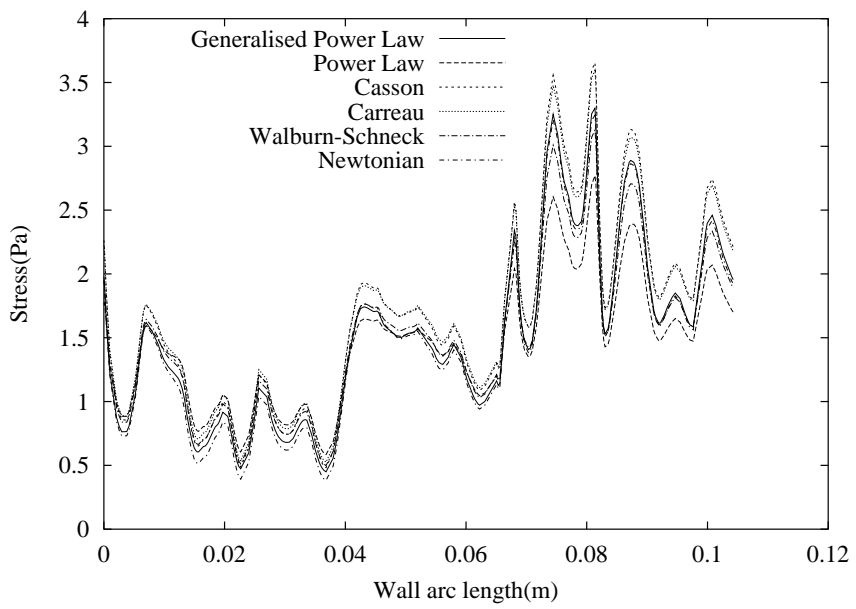

(c) artery D

Figure 11: 


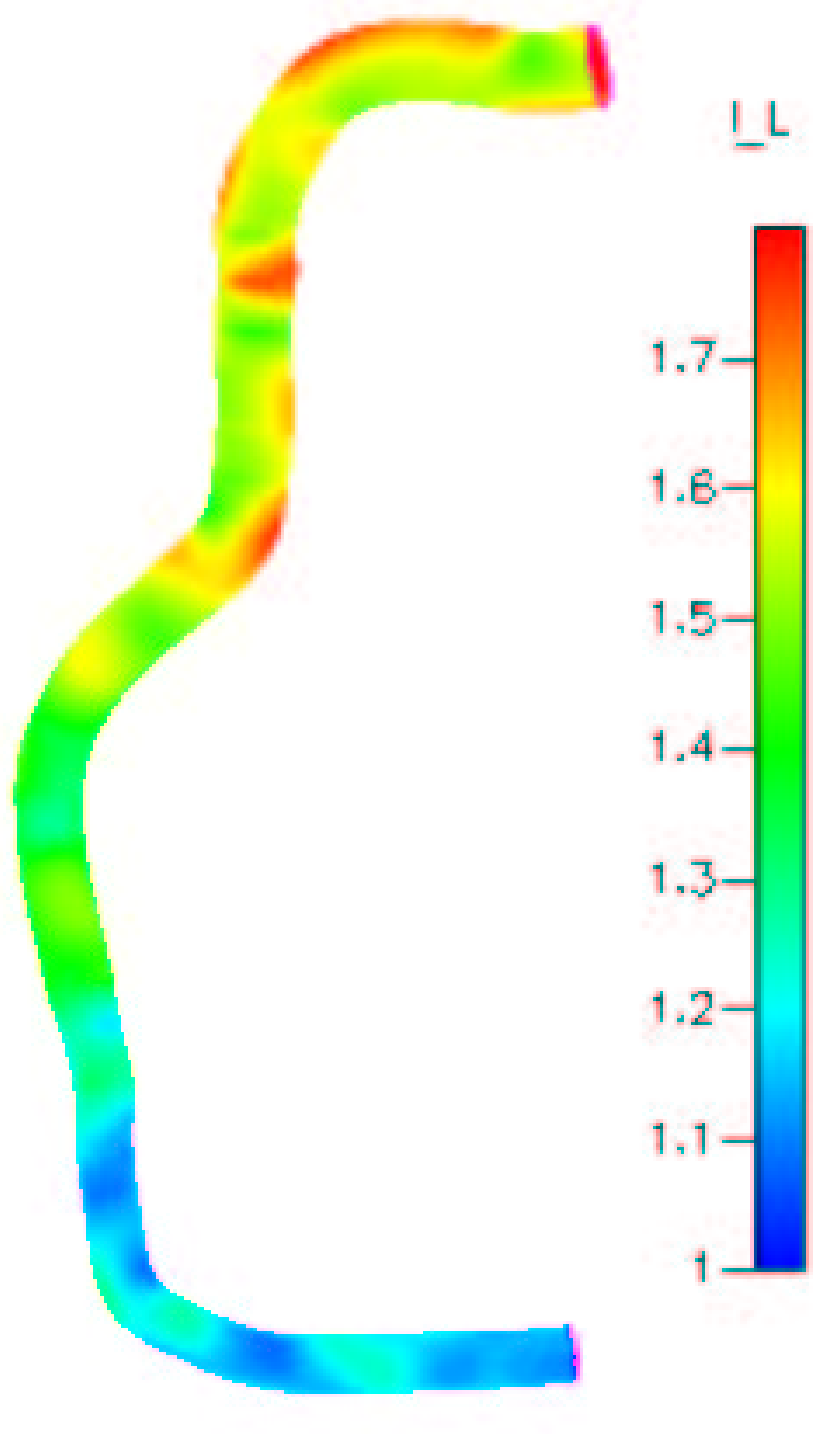

Figure 12: 